\title{
Prevalence and risk factors of smell dysfunction - a comparison between five German population-based studies* $^{*}$
}

\author{
Julia Stögbauer ${ }^{1}$, Kerstin Wirkner ${ }^{2}$, Christoph Engel2,7, Susanne Moebus ${ }^{3}$, \\ Noreen Pundt ${ }^{3}$, Henning Teismann', Markus Löffler ${ }^{2,7}$, Thomas Hummel ${ }^{4}$, \\ Achim Georg Beule ${ }^{5,6}$, Klaus Berger ${ }^{1}$ \\ Rhinology 58: 2, 184 - 193, 2020 \\ https://doi.org/10.4193/Rhin19.181 \\ *Received for publication: \\ ' Institute of Epidemiology and Social Medicine, University of Muenster, Germany \\ April 30, 2019 \\ ${ }^{2}$ LIFE - Leipzig Research Centre for Civilization Diseases, University of Leipzig, Germany \\ Accepted: August 7, 2019 \\ ${ }^{3}$ Centre for Urban Epidemiology, Institute of Medical Informatics, Biometry and Epidemiology, University Hospitals Essen, Germany \\ ${ }^{4}$ Clinic of Otorhinolaryngology, University Hospital Dresden, University of Dresden, Germany \\ 5 Clinic of Otorhinolaryngology, University Hospital Muenster, University of Muenster, Germany \\ ${ }^{6}$ Department of Otorhinolaryngology, University Clinic Greifswald, University of Greifswald, Germany \\ Institute for Medical Informatics, Statistics, and Epidemiology (IMISE),University of Leipzig, Germany
}

\begin{abstract}
Background: The prevalence of olfactory impairment increases with age and is known to be an early sign of different neurodegenerative diseases. Only few population-based studies examined the prevalence of olfactory impairment and comparisons across studies are scarce. Aim of this analysis was to compare the prevalence and determinants of normosmia across five populationbased studies in Germany.
\end{abstract}

Methodology: Data from five population-based, cross-sectional studies were included. They were independently conducted and used the same test system (Sniffin' Sticks Screening 12) to measure olfactory function. This system consists of 12 odor-dispensing felt-tip pens; the task is a forced-choice selection among four alternative odors per pen. Sociodemographic information and comorbidities were assessed in face-to-face interviews. Univariate, descriptive statistics and multivariable logistic regression models stratified by study, were performed to determine risks, i.e. prevalence odds ratios, associated with olfactory function.

Results: The prevalence of normosmic participants varied considerably across studies. Olfactory function was lower in men, decreased with age, and increased with higher education. Several individual comorbidities and a comorbidity index were associated with olfactory dysfunction. Recognition performance for three of the 12 pens was especially low in all studies.

Conclusion: Four factors, well known to describe population composition, contribute to explain differences in the prevalence of olfactory function between studies when the same test system is used. Our results indicate that comorbidities and educational level should always be considered when test systems based on smell recognition are used in population-based studies.

Key words: epidemiologic measurements, health status, olfaction disorders, smell, quality of life

\section{Introduction}

Olfactory function has a remarkable impact on our daily living. Its impairment can result in a considerable decrease in quality of life (1) and is associated with depression ${ }^{(2)}$. A decline in or a loss of olfaction is also related to neurodegenerative disease, i.e. it can be an early sign of Parkinson's and Alzheimer's disease ${ }^{(3-5)}$, years before clinical symptoms occur. Permanent olfactory loss has even been shown to be a powerful predictor of five-year mortality ${ }^{(6)}$. Previous studies have shown that the prevalence of olfactory dysfunction in the general population is considerably 
high ${ }^{(7-9)}$. But despite its influence on the individual well-being and human health olfactory function is rarely assessed in population-based studies ${ }^{(10)}$. The most common risk factors for olfactory dysfunction are older age and male gender ${ }^{(11-13)}$. Current smoking ${ }^{(14)}$ is also known to increase the risk for olfactory impairment; the same holds true for lower social status and specific disorders such as hepatitis B or nonotolaryngological cancers ${ }^{(12,15,16)}$.

There are many different methods to assess olfactory function, ranging from simple self-reports on the one end to the measurement of olfactory event-related potentials on the other end of the method spectrum ${ }^{(17)}$. Among this range of methods, psychophysical olfactory assessment tests are widely applied. Such tests are easy to administer and have been proven to be more reliable than the self-assessment tests ${ }^{(18)}$. Most tests are based on odor identification tasks such as the widely used University of Pennsylvania Smell Identification Test UPSIT ${ }^{(19)}$ or the Connecticut Chemosensory Clinical Research Center Test CCCRC test ${ }^{(20)}$ that adds an olfactory threshold test. Another alternative, the Sniffin' Sticks test, combines identification of different odorants and threshold testing ${ }^{(21)}$. This test has been established in Germany ${ }^{(22-24)}$ and later been adapted to various countries worldwide ${ }^{(25-28)}$. These adaptions consider cultural differences concerning the particular range of common odors. The test's odor identification part - the 'Sniffin' Sticks Screening 12'- is a fast and easy screening method to characterize the participants' olfactory performance as either anosmic, hyposmic or normosmic. Given the wide range of assessment methods, comparisons of the prevalence of olfactory impairment between studies are scarce. Thus, the question whether it is possible to compare olfactory performance in different populations that all used the same testing method has rarely been addressed. Even with the use of the same system, differences in application, technical changes over time, and true differences in socioeconomic and other individual characteristics might contribute to prevalence differences.

Aim of this analysis was to compare the prevalence of normosmia across five large population-based studies in Germany that all used the same test system. We considered differences in application methods and estimated the magnitudes of risk factors for the loss of olfactory function.

\section{Materials and methods}

\section{Study cohorts}

Five independently conducted German studies collaborated in this analysis. All studies were population-based, i.e. initially, participants were randomly drawn from the local citizen registry. The studies were carried out over an eleven-year period between 2003 and 2014. In all studies assessments using the Sniffin' Sticks Screening 12 test had been terminated and results of the Sniffin Sticks assessments had been published for the
Dortmund Health Study, the Heinz Nixdorf Recall Study, the BiDirect-Study and the Life-Adult-Study ${ }^{(14,29-31)}$.

The BiDirect study (BiDirect) ${ }^{(32)}$ examines the relationship between depression and (sub-)clinical arteriosclerosis. Planned as a long-term study, BiDirect includes three cohorts and four personal examinations over a period of 12 years. Cohort 1 consisted of 998 patients hospitalized for an acute episode of depression recruited in seven different psychiatric institutions in and around the city of Münster. Cohort 2 included 347 patients with manifest cardiovascular disease, recruited in four different cardiology departments and rehabilitation clinics in the same area. Cohort 3 is the reference cohort and included 911 community-dwelling adults randomly drawn from the population register of Münster. This analysis included results of the cohort 3 baseline assessment (2010 to 2013). The olfaction examination was completed by 909 persons in this cohort.

The Dortmund Health Study (DHS) ${ }^{(14)}$ was carried out between 2003 and 2004 in order to assess prevalences, risk factors, and consequences of different chronic diseases. A sex-stratified random sample aged 25-74 years was drawn from the population register of the city of Dortmund. Of the 2291 responders, 979 persons merely answered a questionnaire; the remaining 1312 persons participated in a face-to-face interview and different examinations, including tests of sensory performance. Among the latter group, 1230 persons completed the 'Sniffin Sticks Screening 12' (SnSt12) test.

The population-based Heinz Nixdorf Recall Study $(\mathrm{HNR})^{(33)}$ is a prospective cohort study assessing different new imaging and non-imaging techniques for their ability to predict cardiovascular events. The study included one baseline (2000-2003) and two follow-up examinations (2006-2008/ 2011-2014). 4814 subjects randomly drawn from the city registries of Bochum, Essen, and Mühlheim, three cities in the west of Germany, participated in the baseline examination. The Sniffin' Sticks Screening 12 test was included in the second follow up visit in which 3097 individuals participated. In the latter group, 2935 participants completed the SnSt12 test.

The LIFE-Adult-Study (34,35), conducted between 2011 and 2014, assessed the prevalences, early onset biomarkers, genetic predispositions, and the roles of lifestyle factors for major civilization diseases. The initial target population included 10000 persons aged 40-79 years, with a subset of 400 participants aged 18-39 years, and represented a gender -stratified random sample of the residents in the city of Leipzig. 7267 persons completed the SnSt12 test.

The German National Cohort (NAKO) ${ }^{(36)}$ aims to investigate the effects of life-style, occupation and environment, social and psychosocial factors, and genetic predisposition on disease development. Conducted in 18 study centers across Germany, this large-scale study will include 200000 Germans. The baseline assessment is ongoing since October 2014, and will end in 2019, 
Table 1. Characteristics of the participants according to study.

\begin{tabular}{|c|c|c|c|c|c|}
\hline & $\begin{array}{c}\text { BiDirect } \\
n=911\end{array}$ & $\begin{array}{c}\text { DHS } \\
n=1230\end{array}$ & $\begin{array}{c}\text { HNR } \\
n=2934\end{array}$ & $\begin{array}{c}\text { LIFE } \\
n=7267\end{array}$ & $\begin{array}{c}\text { NAKO Pr2 } \\
n=1518\end{array}$ \\
\hline \multicolumn{6}{|l|}{ Sociodemographic factors } \\
\hline Age range (years) & $35-66$ & $26-74$ & $55-86$ & $19-80$ & $20-71$ \\
\hline Age, mean (years) & 52.8 & 52.13 & 68.59 & 57.07 & 52.08 \\
\hline Women, \% & 50.8 & 53.3 & 51.2 & 52.1 & 54.0 \\
\hline High level of education, $\%$ & 48.2 & 29.8 & 24.1 & 34.5 & 45.5 \\
\hline \multicolumn{6}{|l|}{ Smoking status } \\
\hline Never-smoker, \% & 41.6 & 50.3 & 43.6 & 48.1 & 45.2 \\
\hline Ex-smoker, \% & 37,9 & 35.1 & 43.8 & 29.5 & 33.4 \\
\hline Smoker, \% & 20.5 & 14.7 & 12.6 & 22.4 & 21.4 \\
\hline \multicolumn{6}{|l|}{ Comorbidities } \\
\hline History of cancer, \% & 7.0 & 4.6 & 18.2 & 9.8 & 6.9 \\
\hline Known diabetes, \% & 3.5 & 7.5 & 14.9 & 10.5 & 6.5 \\
\hline History of heart attack, \% & 1.9 & 3.7 & 6.7 & 2.2 & 1.8 \\
\hline Known hypertension, \% & 27.1 & 34.9 & 73.9 & 43.0 & 33.1 \\
\hline History of stroke, \% & 1.4 & 2.1 & 3.1 & 2.3 & 1.6 \\
\hline Comorbidity score $\geq 1, \%$ & 33.3 & 39.4 & 79.4 & 49.4 & 39.9 \\
\hline \multicolumn{6}{|l|}{ Smell function } \\
\hline Number of correctly recognizedsticks, mean & 10.6 & 10.4 & 9.0 & 9.9 & 10.1 \\
\hline Prevalence of normosmia, \% & 83.3 & 79.0 & 46.6 & 67.4 & 74.2 \\
\hline
\end{tabular}

BiDirect: BiDirect study; DHS: Dortmund Health Study; HNR: Heinz Nixdorf Recall Study; LIFE: LIFE-Adult-Study; NAKO: German National Cohort.

and will be succeeded by a follow-up examination. Prior to the NAKO baseline examination several pretests evaluating logistics and specific examination methods had been performed. They were already conducted in individuals randomly drawn from the local city registries. This analysis uses data from the NAKO Pretest 2 (NAKO Pr2), which was conducted in 2012, and which included 2904 participants among whom 1518 completed the SnSt12 test.

\section{Olfactory measures}

In all five studies, olfactory function was assessed with the 'Sniffin Sticks Screening 12' (Burghart, Wedel, Germany). This odor identification test utilizes 12 odor-dispensing felt-tip pens (35). During examination, an odor pen was held in front of both nostrils for 3 to 4 seconds. After smelling, the participants had to identify the presented odor by making a choice from four visually presented answer alternatives (forced-choice). 10-12 correct answers defined the subject as 'normosmic', 7-9 correct answers as 'hyposmic' and 0-6 correct answers as 'functionally anosmic' (further termed 'anosmic'). This stratification is based on the official instruction for original report of the Sniffin' Sticks Screening 12 test (35). For this analysis, only data of participants, who completed the test, were used. Persons who missed or did not name one or more of the 12 odors were excluded.

\section{Risk factor assessment}

A common set of five different comorbidities, i.e. histories of diabetes, cancer, heart attack, hypertension, and stroke, was assessed in each study. These were based on lifetime physicianassigned diagnoses, which were self-reported by the participant. Level of education reflects the highest level of school education achieved by the participant. Smoking was categorized into never-, ex-, and current smoker (irrespective of smoking duration) in each study.

\section{Statistical analyses}

Statistical analyses were performed using SPSS version 25 (SPSS Inc., Chicago, IL, USA). Continuous variables were described by means and standard deviations, or by medians and ranges, as was appropriate. Categorical variables were compared between groups using chi-square tests. Variables considered for univariate analysis were age, gender, current smoking, education, and five comorbidities (diabetes, cancer, heart attack, hypertension and stroke). A simple comorbidity index was built as the sum of diseases diagnosed in a participant (0-5 diseases) with regard to the comorbidities listed above. This index was subsequently categorized ( 0,1 and 2, 3 or more comorbidities). Multivariable logistic regression models were applied separately for the five studies to analyze the relationship between normosmia (yes-no, i.e. 10 - 12 correctly recognized smells as the dependent variable) and the risk factors age, gender, level of school education (school degree: none, primary school, intermediate degree, high school), smoking status (current smoker yes-no) and the comorbidity index $(0,1$ or $2,3+)$ as independent variables. Nagelkerke $\mathrm{R} 2$ as the coefficient of variation was calculated for each study to compare explained variances across studies. 


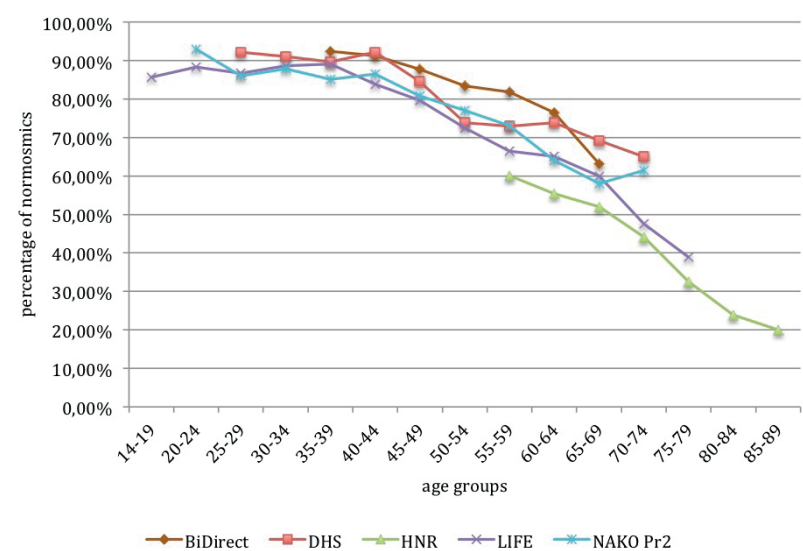

Figure 1. Prevelance of normosmic participants in each study according to age.

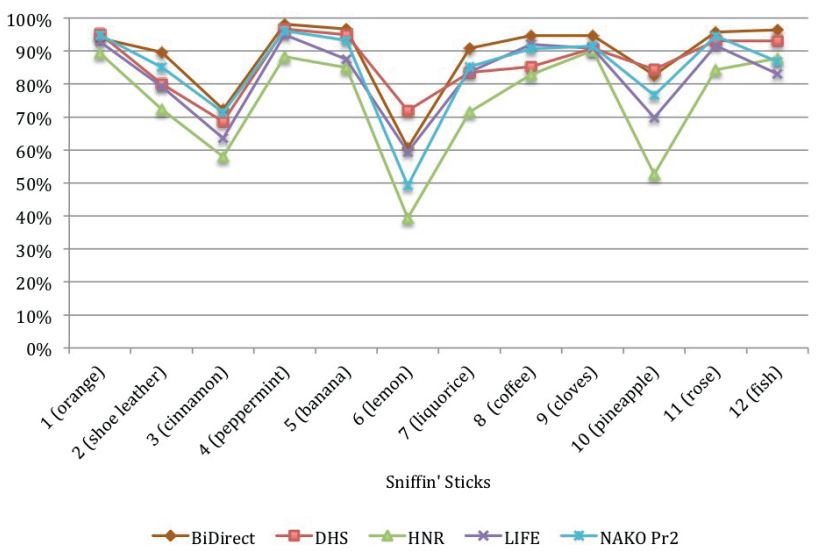

Figure 2. Recognition performance for each Sniffin' Stick according to study.

\section{Results}

Characteristics of the participants are shown in Table 1 stratified by the five studies. A total of 13,825 participants were included. Mean age varied between 52.1 years (NAKO Pr2) and 68.6 years (HNR). The proportion of women was very similar across all studies (50.8 - 54.0\%). The proportion of high school graduates among participants was highest in BiDirect (48.2\%) and lowest in HNR (24.1\%). The proportion of current smokers varied between $12.6 \%$ (HNR) and 20.5\% (BiDirect). Due to the large difference in age, the share of participants with comorbidities differed considerably between studies; for instance, in BiDirect 33.4\% of the participants were affected by at least one of five diseases, compared to $79.4 \%$ in HNR with the oldest study population. Participants in HNR also had the highest prevalence for each of the five diseases individually, which is attributable to their age range of 55-86 years. Across all studies hypertension was the most prevalent disease and stroke the least prevalent one. The overall prevalence of normosmia varied considerably between studies (46.6\% in HNR and $83.3 \%$ in BiDirect). The effect of age in both sexes on the prevalence is shown in Figure 1 and Supplemental Figures $1 \mathrm{~A}$ and 1B. Participants in HNR had the lowest prevalence of normosmia irrespective of age group, while individuals in BiDirect showed the highest prevalence. $\mathrm{A}$ decrease of normosmia below a prevalence of $80 \%$ started in men in most studies in the age group 45 to 49 years; in women, this decline started 5 years later. In Figure 2 the recognition performance for each of the 12 Sniffin' Sticks is shown. Two different patterns can be observed. First, the recognition performance for each individual pen was better in studies observing a high overall prevalence of normosmia. Thus, the positive result was not due to better performance for selected pens. Second, recognition performance was comparably low for three particular pens in all studies. This included pens 3 (cinnamon), 6 (lemon) and 10 (pineapple). In each study these were the three odors with the worst recognition. However, even here recognition followed the prior observed differences in overall prevalence of normosmia between the studies, with lowest levels in HNR.

In univariate analyses (cf. Supplement Table 1) olfactory function was consistently associated with age and gender across studies. Per one year increase in age the odds to be normosmic significantly decreased between 4 (DHS) and 6 (HNR) percent. Men had a 33\% (LIFE) to $45 \%$ (NAKO) lower probability to be normosmic compared to women. In all but two studies (NAKO; BiDirect), a higher school educational level was associated with better olfactory function. Presence of comorbidities generally decreased olfactory performance (Supplemental Table 1). The probability to be normosmic significantly declined by $26 \%$ (DHS) to 35\% (LIFE) for each additional comorbidity in all studies except for NAKO. Within the spectrum of assessed comorbidities histories of diabetes and heart attack were consistently associated with the strongest decline in olfactory function in all five studies. No association was observed between current smoking and olfactory function in three of the five studies.

Table 2 shows the results of the multivariate analysis. The same set of independent variables (age, gender, education, smoking and comorbidity index) was included into the model, which was run stratified by study. In all studies age and gender were significantly associated with normosmia. A high educational level was associated with a higher probability to be normosmic in four studies and significantly in three of them. The comorbidity index was only slightly and inversely associated with normosmia and reached statistical significance only in HNR. Since the set of independent variables was held constant across studies the coefficient of variation (Nagelkerke R2) was comparable between them. It varied between 0.02 and 0.13 , indicating that several additional factors would contribute to the explanation of the variance in normosmia. The largest degree of variance explained by this set of five independent variables was observed in DHS and LIFE.

Olfactory testing was performed over the course of 11 years in 
Table 2. Multivariate analysis of cross-sectional determinants of olfactory function, stratified by study.

\begin{tabular}{|c|c|c|c|}
\hline & OR & $95 \% \mathrm{Cl}$ & $\mathbf{p}$ \\
\hline \multicolumn{4}{|l|}{ BiDirect, n= 911} \\
\hline Age & 0,94 & $0.92-0.97$ & $<0.01$ \\
\hline Currently smoking & 0.70 & $0.45-1.09$ & 0.12 \\
\hline Education & 1.11 & $0.89-1.37$ & 0.35 \\
\hline Gender & 0.68 & $0.47-0.99$ & 0.04 \\
\hline Comorbidity score & 0.94 & $0.68-1.31$ & 0.71 \\
\hline \multicolumn{4}{|l|}{ (Nagelkerke=0,02) } \\
\hline \multicolumn{4}{|l|}{ DHS, n= 1230} \\
\hline Age & 0.97 & $0.95-0.98$ & $<0.01$ \\
\hline Currently smoking & 0.77 & $0.49-1.22$ & 0.27 \\
\hline Education & 1.48 & $1.20-1.82$ & $<0.01$ \\
\hline Gender & 0.59 & $0.44-0.82$ & $<0.01$ \\
\hline Comorbidity score & 0.91 & $0.77-1.08$ & 0.29 \\
\hline \multicolumn{4}{|l|}{$($ Nagelkerke $=0,13)$} \\
\hline \multicolumn{4}{|l|}{$H N R, n=2934$} \\
\hline Age & 0.94 & $0.93-0.95$ & $<0.01$ \\
\hline Currently smoking & 0.65 & $0.51-0.82$ & $<0.01$ \\
\hline Education & 1.16 & $1.06-1.26$ & $<0.01$ \\
\hline Gender & 0.63 & $0.54-0.74$ & $<0.01$ \\
\hline Comorbidity score & 0.86 & $0.76-0.97$ & $<0.01$ \\
\hline \multicolumn{4}{|l|}{ (Nagelkerke $=0,01$ ) } \\
\hline \multicolumn{4}{|l|}{ LIFE, n= 7267} \\
\hline Age & 0.95 & $0.94-0.95$ & $<0.01$ \\
\hline Currently smoking & 0.71 & $0.62-0.81$ & $<0.01$ \\
\hline Education & 1.11 & $1.01-1.21$ & 0.02 \\
\hline Gender & 0.66 & $0.59-0.73$ & $<0.01$ \\
\hline Comorbidity score & 1.02 & $0.93-1.11$ & 0.72 \\
\hline \multicolumn{4}{|l|}{ (Nagelkerke $=0,13)$} \\
\hline \multicolumn{4}{|l|}{ NAKO Pr2, n= 1518} \\
\hline Age & 0.95 & $0.94-0.97$ & $<0.01$ \\
\hline Currently smoking & 1.02 & $0.70-1.49$ & 0.92 \\
\hline Education & 0.88 & $0.66-1.16$ & 0.35 \\
\hline Gender & 0.56 & $0.41-0.76$ & $<0.01$ \\
\hline Comorbidity score & 0.90 & $0.75-1.08$ & 0.25 \\
\hline (Nagelkerke $=0,10)$ & & & \\
\hline
\end{tabular}

Prevalence Odds Ratios are derived from the same model separately applied to each study, $\mathrm{n}$ refers to the number of participants with complete data. In the model normosmia (yes - no) was the dependent variable and the listed five factors the independent explanatory variables.

the included studies. DHS was the earliest (2003-2004) and HNR and LIFE were (both 2011-2014) the latest studies to apply the test. However, in the evaluation of the overall olfactory performance and the recognition of the single odors, no time trend could be observed.

\section{Discussion}

Aim of this analysis was to compare the prevalence of normosmia and factors that influence olfactory function in studies that used the same test to measure smell function. For this purpose all population based studies in Germany that have used the Sniffin' Sticks Screening 12 so far contributed data. A large dif- 
ference in the prevalence of normosmia was observed between studies, despite applying the same test system. Differences in the prevalence were due to different recognition patterns for the set of all 12 pens rather than to better performance for specific single pens. Age and gender were the most important determinants of olfactory dysfunction, with men performing considerably worse than women. Interestingly the level of education was consistently associated with smell function across all studies. While in univariate analysis the number of comorbidities was also related to malfunction, in multivariate analyses this effect was for the larger part explained by age and gender. Three of the 12 pens had consistently worse recognitions across all studies indicating that the combination of odors in the forced choice selection per stick was not optimal. The alternative choices per stick were too similar to be discriminated from the correct smell.

Prior conducted studies used several different smell test systems, making it difficult to compare prevalences of normosmia across studies. Therefore it is of note, that despite using the same test system the five studies included in this analyses had a considerable variation in the prevalence of normosmic participants. Age is the major determinant for a decrease in olfactory performance, as shown in several other surveys ${ }^{(11)}$. In our analyses, olfactory decline started at the age of 45 , which is considerably earlier than observed in UPSIT, where the decrease began at the age of $60{ }^{(19)}$. But it has to be considered that the UPSIT test has a considerable ceiling effect since it is optimized for the investigation of important olfactory disorders. In the study reports for the assessment of normative data for the Sniffin Sticks only healthy (normosmic) individuals were included ${ }^{(10,13)}$. In the studies included in our analyses, however, healthy and not healthy as well as anosmic, hyp- and normosmic individuals are included. Thus, while healthy participants better show the physiologic, age dependent decline, our studies report the age plus disease related decline. This may be the major reason for the earlier decline in our analyses compared to the normative data and the answer to two slightly different questions, decline in healthy people versus decline in the general population. Moreover, women had significantly better test results than men. In line with our results here, an influence of gender on olfaction has been shown in some, but not all previous studies $(8,12,15)$. We observed a strong association of education with olfactory function in all but one of the studies. This is a novel result, which indicates that the principle of odor recognition in a forced choice task, which is the basis of most olfactory function test, is influenced by the level of education (and potentially by cognitive function). Individuals in all studies, except for NAKO $\mathrm{Pr}$, had a higher probability to reach a score in the normosmic range if they had achieved a high degree of education. In this context, higher education likely serves as a proxy for exposition to and better knowledge of a larger variety of odors, especially from the fruit sector. People with a higher education level might have been exposed to more and more diverse odors during their childhood and adult life. An advantage in verbal abilities may also play a role ${ }^{(37)}$. This finding also indicates that the choice of alternative odors per stick is a difficult task. We attribute the observation that the recognition performance for three of the twelve pens was clearly worse across all studies to our impression that the alternative odor in these three cases were too similar to the target odor. This interpretation is supported by the observation that especially for pens 6 (lemon) and 10 (pineapple) the largest variation in the proportion with correct recognition between studies and all pens was found. Thus, in the situation of relative similarity of a forced-choice alternative, one can expect that individuals with the lowest mean educational level would perform worst.

The observation of a considerably lower recognition of these three pens has been reported before, by one of the included studies ${ }^{(38,39)}$. According to the test manufacturer, there have been no technical changes in the production of the Sniffin' Sticks during the relevant time period of eleven years which rules out other potential alternative explanation. While in previous reports ${ }^{(8,9,15,20,28)}$ differences in the prevalence were most likely attributable to the use of different test systems for olfactory function, this argument cannot be maintained in our analyses. Given the observed effects of the four factors age, gender, education, and comorbidity burden in the majority of the included studies, it seems that the population composition, which is traditionally described by these four factors, considerably contributes to the differences in the observed prevalences of normosmia.

A further approach is to question the answer alternatives for the three odors. At some point, odors may have been too difficult to discriminate, for example in the case of answer possibilities 'grapefruit' and 'citrus' in case of pen 6. Compared to the UPSIT, the forced-choice alternatives of the Sniffin' Sticks Screening 12 test contain response options that are generally more similar and, thus, more difficult to discriminate. For instance the odor 'orange' (stick one) had to be identified from a choice of four sweet fruits (orange, blackberry, strawberry and pineapple), similar to the odor 'pineapple' (stick ten) that had to be discriminated from plum, peach and pear. This may explain that in the included studies the decrease in olfactory performance started at a considerably younger age than stated in UPSIT. Furthermore, the test results can be expected to be influenced by prior experience. Implementation of the Sniffin' Sticks in both, clinical ENT and neurology units, may increase the proportion of subjects in contact with this test in more recent studies. Also, a higher educational level may be associated with better memory capacity ${ }^{(40,41)}$.

Our analysis has considerable strengths and some weaknesses. First, we included all population-based studies that have used 
the Sniffin' Sticks Screening 12 test so far; thus, our analysis is the largest comparison of olfactory function between studies in Germany. Second, in all studies, the proportions of women and men were comparable, and the age of the participants spanned a wide range. Third, all studies used the same, easy-to-apply Sniffin' Sticks Screening 12 test system and assessed sociodemographic characteristics and comorbidities in a similar way. However, there were slight differences in the performed applications of the Sniffin'Sticks Screening 12 test among the five studies. For example, gloves were used in HNR and LIFE, but not in BiDirect, DHS, and NAKO Pr2. In HNR, the four answer alternatives were shown to the participant only after the pen was presented for smelling; in the other four studies, the list was shown before pen exposure. With respect to the former, there is some evidence for worse test performance ${ }^{(42)}$ if presented afterwards. Recognition performance for three of the 12 pens was especially low in all studies. This may give rise to a discussion about a change of distractors for these three odors. Finally, some differences in the prevalences of comorbidities between studies existed, resulting in only few affected individuals in some studies.

\section{Conclusion}

In a comparison of five large, population-based studies, we found differences in the prevalence of normosmia within each age and gender specific stratum, despite the use of the same system to test smell function across studies. Four factors, which are well- known to describe population composition, i.e. age, gender, educational level and comorbidity burden, considerably contributed to the explanation of prevalence differences. Our results also indicate that educational level should always be considered when test systems based on smell recognition are used in population- or patient-based studies.

\section{Acknowledgements}

The BiDirect Study is funded by the German Federal Ministry of Education and Research (BMBF, (01ER0816 and 01ER1506).

Data collection in the Dortmund Health Study was supported by the German Migraine \& Headache Society and by unrestricted grants of equal share from Almirall, Astra Zeneca, Berlin Chemie, Boehringer, Boots Health Care, Glaxo-Smith-Kline, Janssen Cilag, McNeil Pharma, MSD Sharp \& Dohme and Pfizer to the University of Muenster. The LIFE Adult study was supported by Leipzig Research Centre for Civilization Diseases LIFE), an organizational unit affiliated to the Medical Faculty of the University of Leipzig, Germany. LIFE is funded by means of the European Union, by the European Regional Development Fund (ERDF) and by funds of the Free State of Saxony within the framework of the excellence initiative (project numbers 713-241202, 713-241202, $14505 / 2470,14575 / 2470$ ). We wish to thank the citizens of the City of Leipzig for their willingness to participate in the study. We also thank the team of the LIFE-Adult-Study centre for their skilful technical assistance. The Heinz Nixdorf Recall study was funded by the Heinz Nixdorf Foundation, Germany [Chairman: M. Nixdorf ; Past Chairman: Dr. jur. G. Schmidt (deceased)]. The HNR was also supported by grants from the German Research Council [Deutsche Forschungsgemeinschaft (DFG); ER 155/6-1, ER 155/6-2, SI 236/8-1, and SI 236/9-1] and the Kulturstiftung Essen, Germany. The NAKO Pretest 2 was funded by the German Federal Ministry of Education and Research (01ER1001D).

\section{Authorship contribution}

Contributions to the conception/design of each of the five participating study, their data acquisition and analysis: DHS (TH, $\mathrm{KB})$, LIFE (KW, CE, ML), BiDirect (HT, KB), RECALL (SM, DP), NAKO Pre2 (KB, $A B)$.

Drafting the manuscript (JS, KB) and revising it for important intellectual content ( $T H, K W, C E, M L, H A T, S M, D P, A B)$.

Approval of the final version (TH, KW, CE, ML, HAT, SM, DP, AB, JS, $\mathrm{KB})$.

\section{Conflict of interest}

On behalf of all authors, the corresponding author states that there is no conflict of interest.

\section{References}

1. Croy I, Nordin S, Hummel T. Olfactory disorders and quality of life--an updated review. Chem Senses. 2014; 39: 185-194.

2. Croy I, Symmank A, Schellong J, et al. Olfaction as a marker for depression in humans. J Affect Disord. 2014; 160: 80-86.

3. Hoyles K, Sharma JC. Olfactory loss as a supporting feature in the diagnosis of Parkinson's disease: a pragmatic approach. J Neurol. 2013; 260: 2951-2958.

4. Marine N, Boriana A. Olfactory markers of depression and Alzheimer's disease. Neurosci Biobehav Rev. 2014; 45: 262-270.

5. Hummel T, Whitcroft KL, Andrews $P$ et al. Position paper on olfactory dysfunction.
Rhinology. 2016; 56: 1-30.

6. Pinto JM, Wroblewski KE, Kern DW, Schumm LP, McClintock MK. Olfactory dysfunction predicts 5-year mortality in older adults. PLoS One. 2014; 9: e107541.

7. Landis BN, Hummel T. New evidence for high occurrence of olfactory dysfunctions within the population. Am J Med. 2006; 119: 91-92.

8. Bramerson A, Johansson L, Ek L, Nordin S, Bende M. Prevalence of olfactory dysfunction: the skovde population-based study. Laryngoscope. 2004; 114: 733-737.

9. Schubert CR, Cruickshanks KJ, Fischer ME et al. Olfactory impairment in an adult population: the Beaver Dam Offspring Study.
Chem Senses. 2012; 37: 325-234.

10. Oleszkiewicz A, Schriever VA, Croy I, Hahner A, Hummel T. Updated Sniffin' Sticks normative data based on an extended sample of 9139 subjects. Eur Arch Otorhinolaryngol. 2019; 276: 719-728.

11. Doty $R L$, Kamath $V$. The influences of age on olfaction: a review. Front Psychol. 2014; 5: 20 .

12. Larsson M, Finkel D, Pedersen NL. Odor identification: influences of age, gender, cognition, and personality. J Gerontol B Psychol Sci Soc Sci. 2000; 55: 304-310.

13. Hummel T, Kobal G, Gudziol H, Mackay-Sim A. Normative data for the "Sniffin' Sticks" including tests of odor identification, odor 
discrimination, and olfactory thresholds: an upgrade based on a group of more than 3,000 subjects. Eur Arch Otorhinolaryngol. 2007; 264: 237-43.

14. Vennemann MM, Hummel T, Berger K. The association between smoking and smell and taste impairment in the general population. J Neurol. 2008; 255: 1121-1126.

15. Lee WH, Wee JH, Kim DK et al. Prevalence of subjective olfactory dysfunction and its risk factors: korean national health and nutrition examination survey. PLoS One. 2013 8: e62725.

16. Landis BN, Konnerth CG, Hummel T. A study on the frequency of olfactory dysfunction. Laryngoscope. 2004; 114(10): 1764-1769.

17. Nguyen DT, Rumeau C, Gallet P, Jankowsk R. Olfactory exploration: State of the art. Eur Ann Otorhinolaryngol Head Neck Dis. 2016; 133: 113-118

18. Nguyen DT, Nguyen-Thi PL, Jankowski R. How does measured olfactory function correlate with self-ratings of the sense of smell in patients with nasal polyposis? Laryngoscope. 2012; 122: 947-952.

19. Doty RL, Shaman P, Kimmelman CP, Dann MS. University of Pennsylvania Smell Identification Test: a rapid quantitative olfactory function test for the clinic. Laryngoscope. 1984; 94 :176-178.

20. Heywood PG, Costanzo RM. Identifying normosmics: a comparison of two populations. Am J Otolaryngol. 1986; 7:194-199.

21. Rumeau C, Nguyen DT, Jankowski R. How to assess olfactory performance with the Sniffin' Sticks test((R)). Eur Ann Otorhinolaryngol Head Neck Dis. 2016; 133: 203-206.

22. Hummel T, Sekinger B, Wolf SR, Pauli E, Kobal G. 'Sniffin' sticks': olfactory performance assessed by the combined testing of odor identification, odor discrimination and olfactory threshold. Chem Senses. 1997; 22 39-52.

23. Reden J, Draf C, Frank RA, Hummel T. Comparison of clinical tests of olfactory function. Eur Arch Otorhinolaryngol. 2016; 273: 927-931.

24. Haehner A, Mayer AM, Landis BN et al. High test-retest reliability of the extended version of the "Sniffin' Sticks" test. Chem Senses. 2009; 34: 705-711.
25. Antsov E, Silveira-Moriyama L, Kilk S et al. Adapting the Sniffin' Sticks olfactory test to diagnose Parkinson's disease in Estonia. Parkinsonism Relat Disord. 2014; 20: 830833.

26. Konstantinidis I, Printza A, Genetzaki S Mamali K, Kekes G, Constantinidis J. Cultural adaptation of an olfactory identification test: the Greek version of Sniffin' Sticks. Rhinology. 2008; 46: 292-296.

27. Sorokowska A, Hummel T. Polish version of the Sniffin' Sticks Test - adaptation and normalization. Otolaryngol Pol. 2014; 68: 308-314.

28. Oleszkiewicz A, Taut M, Sorokowska A, Radwan A, Kamel R, Hummel T. Development of the Arabic version of the "Sniffin' Sticks" odor identification test. Eur Arch Otorhinolaryngol. 2016; 273: 1179 1184.

29. Tebrugge S, Winkler A, Gerards D et al. Olfactory Function is Associated with Cognitive Performance: Results of the Heinz Nixdorf Recall Study. J Alzheimers Dis. 2018; 63: 319-29.

30. Yahiaoui-Doktor M, Luck T, Riedel-Heller SG, Loeffler M, Wirkner K, Engel C. Olfactory function is associated with cognitive performance: results from the population-based LIFE-Adult-Study. Alzheimers Res Ther. 2019; 11: 43

31. Khil L, Rahe C, Wellmann J, Baune BT, Wersching $\mathrm{H}$, Berger K. Association between major depressive disorder and odor identification impairment. J Affect Disord. 2016; 203 :332-8

32. Teismann $H$, Wersching $H$, Nagel $M$ et al. Establishing the bidirectional relationship between depression and subclinical arteriosclerosis--rationale, design, and characteristics of the BiDirect Study. BMC Psychiatry. 2014: 14: 174.

33. Kara K, Geisel MH, Mohlenkamp S, et al. B-type natriuretic peptide for incident atrial fibrillation-The Heinz Nixdorf Recall Study. J Cardiol. 2015; 65: 453-458.

34. Loeffler M, Engel C, Ahnert $P$ et al. The LIFEAdult-Study: objectives and design of a population-based cohort study with 10,000 deeply phenotyped adults in Germany. BMC Public Health. 2015; 15: 691.

35. Hummel T, Konnerth CG, Rosenheim K
Kobal G. Screening of olfactory function with a four-minute odor identification test: reliability, normative data, and investigations in patients with olfactory loss. Ann Otol Rhinol Laryngol. 2001; 110: 976-981.

36. Consortium GNC. The German National Cohort: aims, study, design and organization. Eur J Epidemiol. 2014; 29: 371-382.

37. Larsson M, Lovden M, Nilsson LG. Sex differences in recollective experience for olfactory and verbal information. Acta Psychol (Amst). 2003; 112: 89-103.

38. Sorokowska A, Albrecht E, Haehner A, Hummel T. Extended version of the "Sniffin' Sticks" identification test: test-retest reliability and validity. J Neurosci Methods. 2015; 243: 111-114.

39. Hinz A, Luck T, Riedel-Heller SG et al. Olfactory dysfunction: properties of the Sniffin' Sticks Screening 12 test and associations with quality of life. Eur Arch Otorhinolaryngol. 2018.

40. Hsieh JW, Keller A, Wong M, Jiang RS, Vosshall LB. SMELL-S and SMELL-R: Olfactory tests not influenced by odor-specific insensitivity or prior olfactory experience. Proc Natl Acad Sci U S A. 2017: 114: 11275-11284

41. Thomas-Danguin $T$, Sinding C, Romagny $S$ et al. The perception of odor objects in everyday life: a review on the processing of odor mixtures. Front Psychol. 2014; 5: 504

42. Sorokowska A, Albrecht E, Hummel T. Reading first or smelling first? Effects of presentation order on odor identification. Atten Percept Psychophys. 2015; 77: 731736.

Klaus Berger MD, MSC, MPH Institute of Epidemiology and Social

Medicine

University of Münster

Domagkstr. 3

48149 Münster

Germany

E-mail: bergerk@uni-muenster.de

ORCID: 0000-0001-8966-3684

This paper contains supplementary materials online: at www.rhinologyjournal.org 
Supplementary Figures

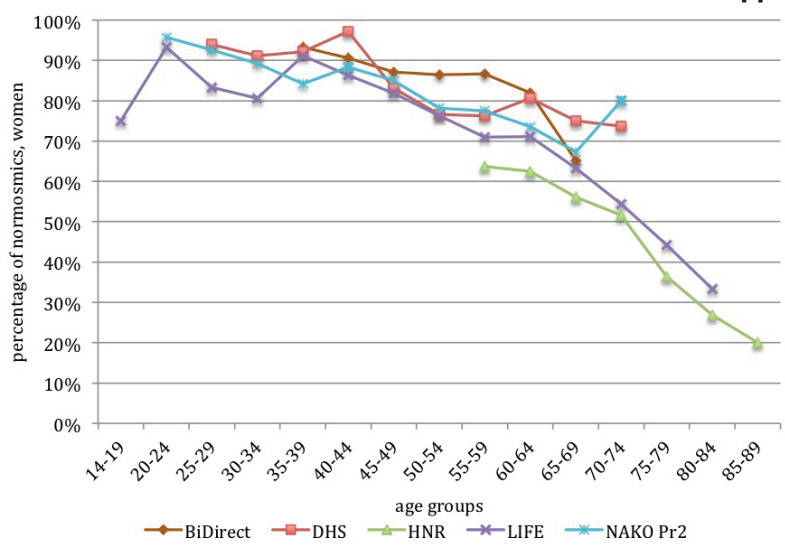

Supplementary Figure 1A. Prevelance of normosmic women in each study according to age.

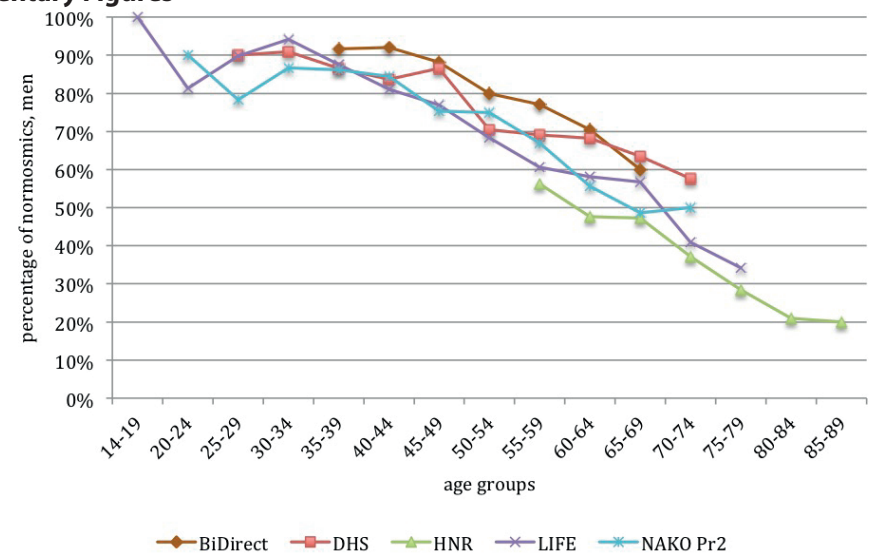

Supplementary Figure 1B. Prevelance of normosmic men in each study according to age.

Supplemental Table 1. Univariate analysis of the association between normosmia and sociodemographic variables and assessed comorbidities within each study.

\begin{tabular}{|c|c|c|c|}
\hline & OR & $95 \% \mathrm{Cl}$ & $\mathbf{p}$ \\
\hline \multicolumn{4}{|l|}{ BiDirect, n= 911} \\
\hline Age & 0.94 & $0.92-0,96$ & $<0.01$ \\
\hline Currently smoking & 0.77 & $0.51-1.18$ & 0.23 \\
\hline Education & 1.07 & $0.87-1.32$ & 0.50 \\
\hline Gender & 0.70 & $0.49-1.00$ & 0.05 \\
\hline Cancer, $n=64$ & 0.84 & $0.44-1.62$ & 0.60 \\
\hline Diabetes, $n=32$ & 0.30 & $0.14-0.63$ & $<0.01$ \\
\hline Heart attack, $n=17$ & 0.32 & $0.11-0.91$ & 0.03 \\
\hline Hypertension, n= 247 & 0.86 & $0.58-1.26$ & 0.43 \\
\hline Stroke, $n=13$ & 1.10 & $0.24-5.03$ & 0.90 \\
\hline Comorbidity score & 0.71 & $0.53-0.96$ & 0.02 \\
\hline \multicolumn{4}{|l|}{ DHS, $n=1230$} \\
\hline Age & 0.96 & $0.95-0.97$ & $<0.01$ \\
\hline Currently smoking & 1.02 & $0.67-1.57$ & 0.91 \\
\hline Education & 1.67 & $1.41-1.98$ & $<0.01$ \\
\hline Gender & 0.57 & $0.43-0.75$ & $<0.01$ \\
\hline Cancer, $\mathrm{n}=56$ & 0.72 & $0.9-1.32$ & 0.28 \\
\hline Diabetes, $n=92$ & 0.55 & $0.34-0.87$ & 0.01 \\
\hline Heart attack, n= 46 & 0.48 & $0.26-0.89$ & 0.02 \\
\hline Hypertension, $n=429$ & 0.62 & $0.47-0.82$ & 0.00 \\
\hline Stroke, $n=26$ & 0.42 & $0.19-0.93$ & 0.03 \\
\hline Comorbidity score & 0.79 & $0.70-0.89$ & $<0.01$ \\
\hline \multicolumn{4}{|l|}{ HNR, n= 2934} \\
\hline Age & 0.94 & $0.93-0.95$ & $<0.01$ \\
\hline Currently smoking & 0.84 & $0.68-1.05$ & 0.12 \\
\hline Education & 1.22 & $1.12-1.33$ & $<0.01$ \\
\hline Gender & 0.64 & $0.56-0.4$ & $<0.01$ \\
\hline Cancer, $n=533$ & 0.77 & $0.64-0.94$ & 0.01 \\
\hline
\end{tabular}


OR

Diabetes, $n=437$
Heart attack, $n=197$
Hypertension, $n=2169$
Stroke, $n=91$
Comorbidity score

0.79

0.54

0.72

0.55

0.73

$95 \% \mathrm{Cl}$

$\begin{array}{ll}0.64-0.97 & 0.02 \\ 0.40-0.4 & <0.01 \\ 0.61-0.85 & <0.01 \\ 0.36-0.86 & 0,01 \\ 0.65-0.82 & <0,01\end{array}$

\section{니FE, $n=7267$}

\begin{tabular}{|c|c|c|c|}
\hline Age & 0.95 & $0.94-0.95$ & $<0.01$ \\
\hline Currently smoking & 1.01 & $0.90-1.10$ & 0.82 \\
\hline Education & 1.42 & $1.31-1.54$ & $<0.01$ \\
\hline Gender & 0.67 & $0.61-0.74$ & $<0.01$ \\
\hline Cancer, $\mathrm{n}=711$ & 0.81 & $0.69-0.71$ & 0.01 \\
\hline Diabetes, $n=759$ & 0.49 & $0.42-0.57$ & $<0.01$ \\
\hline Heart attack, n= 159 & 0.49 & $0.36-0.68$ & $<0.01$ \\
\hline Hypertension, n= 3101 & 0.60 & $0.55-0.67$ & $<0.01$ \\
\hline Stroke, $n=163$ & $0, .70$ & $0.42-0.78$ & $<0.01$ \\
\hline Comorbidity score & 0.65 & $0.61-0.74$ & $<0.01$ \\
\hline \multicolumn{4}{|l|}{ NAKO Pr2, n= 1518} \\
\hline Age & 0.95 & $0.94-0.96$ & $<0.01$ \\
\hline Currently smoking & 1.05 & $0.73-1.51$ & 0.79 \\
\hline Education & 0.95 & $0.81-1.12$ & 0.56 \\
\hline Gender & 0.55 & $0.43-0.69$ & $<0.01$ \\
\hline Cancer, $n=68$ & 1.30 & $0.71-2.39$ & 0.40 \\
\hline Diabetes, $n=64$ & 0.61 & $0.36-1.05$ & 0.07 \\
\hline Heart attack, $n=18$ & 0.51 & $0.20-1.34$ & 0.17 \\
\hline Hypertension, n= 326 & 0.93 & $0.69-1.27$ & 0.66 \\
\hline Stroke, $n=16$ & 2.36 & $0.53-10.44$ & 0.26 \\
\hline Comorbidity score & 0.95 & $0.74-1.22$ & 0.69 \\
\hline
\end{tabular}

Prevalence Odds Ratios are derived from a univariate logistic regression model applied to each study. In the model normosmia (yes - no) was the dependent variable and each of the listed factors the independent explanatory variable without further adjustment. 